\title{
Psychological factors predict an unfavorable pain trajectory after hysterectomy: a prospective cohort study on chronic postsurgical pain
}

\author{
Patrícia R. Pinto ${ }^{a, b, \star}$, Teresa McIntyre ${ }^{c}$, Vera Araújo-Soares ${ }^{d}$, Armando Almeida ${ }^{a, b}$, Patrício Costa ${ }^{a, b, e}$
}

\begin{abstract}
Chronic postsurgical pain (CPSP) is a well-recognized potential complication with negative personal, social, and health care consequences. However, limited data exist on CPSP and on the course of pain over time after hysterectomy. Using data from a prospective cohort study on a consecutive sample assessed at 4 time points, presurgery (T1), 48 hours (T2), 4 months (T3), and 5 years postsurgery (T4), we sought to examine women's PSP trajectories using assessments of pain at T3 and T4. In addition, this study aimed to investigate presurgical and postsurgical risk factors associated with an unfavourable pain trajectory (PT). Based on pain data collected at T3 and T4, 3 distinct trajectories of PSP emerged: no CPSP (PT1; $n=88$ ), prolonged PSP (PT2; $n=53$ ), and CPSP (PT3; $n=29$ ). Moreover, reported CPSP prevalence at 5 years was $17.1 \%$. Multinomial logistic regression models controlling for age, presurgical pain, and type of hysterectomy tested for baseline and acute postsurgical predictive variables. Membership in PT2 and PT3 was predicted by presurgical anxiety (odds ratio $[\mathrm{OR}]=1.131, P=0.015 ; \mathrm{OR}=1.175, P=0.009$, respectively), emotional representation of the surgical disease $(\mathrm{OR}=1.155, P=0.034$; $\mathrm{OR}=1.213, P=0.020$, respectively), and pain catastrophizing $(\mathrm{OR}=1.079, P=0.043 ; \mathrm{OR}=1.143, P=0.001$, respectively). Furthermore, acute PSP intensity and frequency determined membership of women in PT3 (OR $=1.211, P=0.033 ; \mathrm{OR}=3.000, P=0.029$, respectively), and postsurgical anxiety $(\mathrm{OR}=1.182, P=0.026)$ also played a key predictive role. This study identified factors that can be easily screened before and after surgery and are amenable to change through carefully designed timely and tailored interventions for women at risk of an unfavorable PSP trajectory posthysterectomy.
\end{abstract}

Keywords: Hysterectomy, Postsurgical pain trajectory, Chronic postsurgical pain, Prospective cohort study, Psychological factors, Acute postsurgical pain

\section{Introduction}

Chronic pain after surgery is a well-recognized potential complication, being acknowledged as a major clinical problem with significant individual, social, and health care costs. ${ }^{51,55,59}$ It is a serious public health issue because surgeries are widely performed, increasing the numbers of those at risk. ${ }^{13,48,55}$ Indeed, the current version of the International Classification of Diseases (ICD-11) for chronic pain categorization already

Sponsorships or competing interests that may be relevant to content are disclosed at the end of this article.

a Life and Health Sciences Research Institute (ICVS), School of Medicine, University of Minho, Braga, Portugal, ' ${ }^{\circ}$ ICVS/3B's_-PT Government Associate Laboratory, Braga/Guimarães, Portugal, ${ }^{c}$ Department of Psychology, College of Education and Behavioral Sciences, Houston Baptist University, Houston, TX, USA, ${ }^{d}$ Institute of Health and Society, Faculty of Medical Sciences, Newcastle University, Newcastle upon Tyne, United Kingdom, ${ }^{e}$ Faculty of Psychology and Education Sciences, University of Porto, Porto, Portugal

*Corresponding author. Address: Life and Health Sciences Research Institute (ICVS), School of Medicine, Campus de Gualtar, University of Minho, Braga 4710057, Portugal. Tel.:351-253-604926; fax: 351-253-604809. E-mail address: patipinto@med.uminho.pt (P.R. Pinto).

Supplemental digital content is available for this article. Direct URL citations appear in the printed text and are provided in the HTML and PDF versions of this article on the journal's Web site (www. painjournalonline.com).

PAIN 159 (2018) 956-967

(C) 2018 International Association for the Study of Pain

http://dx.doi.org/10.1097/j.pain.0000000000001170 proposes chronic postsurgical pain (CPSP) as a new distinct entity among 7 groups of chronic pain disorders. ${ }^{102}$

Chronic PSP was first mentioned in $1998,{ }^{26}$ being highlighted that $22.5 \%$ of patients attending 10 pain clinics pointed surgery as the cause of chronic pain. Despite improvements in knowledge regarding epidemiology and CPSP burden, its underlying mechanisms are not fully understood. ${ }^{47,104}$ However, evidence suggests that CPSP development is multifactorial, rooted in a dynamic and complex interplay among biological, psychosocial, and environmental factors. ${ }^{23,48}$ To increase knowledge, research has focused increasingly on understanding risk factors, in hopes of finding new ways to treat and ultimately prevent CPSP from occurring.

Risk factors are often conceptualized into presurgical, intrasurgical, and postsurgical, embracing variables such as age, surgery type, previous pain, and acute PSP. 51,52,59,62,73 Psychological factors are well-documented predictors for CPSP across different surgeries, ${ }^{2,15,41,44,61,76}$ including anxiety, ${ }^{58,61,99}$ depression, ${ }^{108}$ pain catastrophizing, ${ }^{28,50,56,58,99}$ and optimism. ${ }^{40,82}$ In an attempt to systematize information, VanDenKerkhof et al. ${ }^{104}$ proposed a framework wherein risk factors are organized into 5 domains: demographic, pain, clinical, surgery related, and psychological.

Hysterectomy is the most common gynecologic surgery performed in women in Western countries. ${ }^{85,98}$ In a review including 11 hysterectomy studies, with follow-up times up to 2 years, CPSP was reported by $5 \%$ to $32 \%$ of women. ${ }^{8}$ 
Subsequent studies kept diverging on prevalence rates. These differences were likely due to distinct CPSP definitions, heterogeneity of measurement, and differences in follow-up times; some were $3,{ }^{100} 4,^{6,67,79} 6$ months, ${ }^{80,103} 1^{7,67,100}$ or 2 years. ${ }^{67}$ Simultaneously, risk factors that have been most highlighted were age, ${ }^{67,79}$ presurgical pain, ${ }^{7,67,100,103}$ other pain problems, ${ }^{6,7,67,79}$ and psychological factors. ${ }^{67,79,100,103}$ Within the latter, there was some heterogeneity because of different measures used. Furthermore, acute PSP intensity was more strongly related with CPSP in some hysterectomy studies $^{6,100,103}$ than in others, ${ }^{67,79}$ with one of the studies proposing PSP frequency as a better predictor than intensity. $^{79}$

Although the incidence and risk factors for CPSP have been widely explored, knowledge on the course of PSP over time is limited. Identifying patients who are at risk of an unfavorable pain trajectory $(\mathrm{PT})$, culminating in CPSP, provides a unique opportunity to investigate the transition from acute to chronic pain and is critical to developing a more comprehensive understanding of CPSP and establishing potential targets for psychosocial and clinical interventions.

Therefore, the aims of this study were: (1) to examine PSP trajectory up to 5 years after hysterectomy; (2) to investigate baseline predictors of different pain trajectories; and (3) to further explore the added predictive value of acute postsurgical factors in pain chronification.

\section{Methods}

\subsection{Participants and procedure}

This study was conducted in a central hospital in northern Portugal and approval was granted by the Hospital Ethics Committee. This was a prospective cohort study with longitudinal assessments at 4 time points: 24 hours before surgery (T1), 48 hours (T2), 4 months (T3), and 5 years (T4) after surgery. Assessments were performed between March 2009 and January 2015. A consecutive sample of 203 women, undergoing hysterectomy due to benign causes, was invited to participate in the study and all provided written informed consent. Inclusion criteria were age between 18 and 80 years, and the ability to understand consent procedures and questionnaire materials. Exclusion criteria were the presence of psychiatric or neurologic pathology (eg, dementia) and undergoing hysterectomy due to malign conditions or in emergency setting. Time 1 and T2 assessments took place in hospital, T3 and T4 follow-up assessments were conducted by telephone interview. Inclusion in each assessment point and the reasons for loss to T2, T3, and T4 are shown in a flowchart (Fig. 1). The final sample is comprised of 170 women (retention rate: 83.7\%; age: $M=50.8$; $S D=9.03$; minimum $=35$; maximum $=76$ ) with assessments performed at T1, T2, T3, and T4. The 33 women lost to follow-up, from T1 to $\mathrm{T} 4$, did not differ significantly from the women evaluated over 5 years in terms of baseline demographic, psychological, and clinical characteristics, namely on surgical, anesthetic, and analgesic issues.

Data related to this sample have been described in 4 previous publications reporting predictors of acute PSP, ${ }^{75,76}$ predictors of rescue analgesia administration, ${ }^{78}$ and predictors of persistent PSP 4 months after hysterectomy. ${ }^{79}$ The present work is the first to report the long-term outcomes in this sample, 5 years posthysterectomy, including the 4 assessment points.

\subsection{Data collection}

\subsubsection{Presurgical assessment-24 hours before surgery (T1)}

At hospital admission, and to get a baseline evaluation of women, the Portuguese versions of the following questionnaires were administered, in a face-to-face interview, by a trained health psychologist.

\subsubsection{Sociodemographic Questionnaire}

Included questions on age, height, weight, education, residence, marital status, professional status, household, and parity.

\subsubsection{Clinical Questionnaire}

Enquired about previous pain, either related to the cause of surgery or due to other causes, previous surgeries, menopause status, diagnosis/indication for hysterectomy and disease onset, uterus height and weight, as well as the use of psychotropic drugs (anxiolytics and antidepressants).

\subsubsection{The Brief Pain Inventory-short form}

Used among women reporting presurgical pain (related with the disease underlying the surgery). ${ }^{24}$ It measured pain location in the body; pain intensity on an 11-point numerical rating scale (NRS; 0 represents "no pain" and 10 the "worst pain imaginable"); analgesic intake; perception of analgesic relief; and pain interference with daily life on an 11-point NRS $(0=$ "does not interfere" and 10 = "completely interferes") in distinct dimensions (general activity, mood, walking, work, relations with others, sleep, and enjoyment of life). Higher scores represent higher levels of pain interference. In this study, the internal consistency reliability ${ }^{27}$ for the pain interference subscale scores was high ( $\alpha$ $=0.90)$.

\subsubsection{The Hospital Anxiety and Depression Scale}

Comprised by 2 subscales used to measure anxiety and depression through 7 items each. ${ }^{110}$ Subscale scores range from 0 to 21 and result from the sum of each item (Likert scale ranging from 0 to 3). Higher scores correspond to higher levels of anxiety and depression. In the current sample, internal consistency reliability was adequate for both anxiety (T1: $\alpha=0.77 ; \mathrm{T} 2: \alpha$ $=0.84)$ and depression (T1: $\alpha=0.81)$.

\subsubsection{The Surgical Fear Questionnaire}

Used to evaluate specific surgical fears through 8 items aggregated in 2 subscales, "fear of immediate consequences of surgery" ( $\alpha=0.77 ; 4$ items) and "fear of long-term consequences of surgery" ( $\alpha=0.75 ; 4$ items). ${ }^{101}$ Each item score ranges from 0 to 10; item scores are summed to calculate each total subscale score. Subscale scores range between 0 and 40, with higher values indicating higher levels of fear.

\subsubsection{The Revised Illness Perception Questionnaire}

Used to assess patient beliefs about the underlying disease that lead to surgery, is comprised by 7 dimensions. ${ }^{68}$ In the current study, and with the aim of diminishing participant burden, a psychometrically shortened version ${ }^{77,79}$ was used, with 3 items composing each one of the 7 subscales: "timeline acute/chronic" ( $\alpha=0.79$; eg, "My illness will last for a long time"); "timeline cyclical" ( $\alpha=0.74$; eg, "My symptoms come and go in cycles"); "consequences" ( $\alpha=0.56$; eg, "The disease underlying surgery has major consequences on my life"); "personal control" $(\alpha=0.53$; eg, "I have the power to 


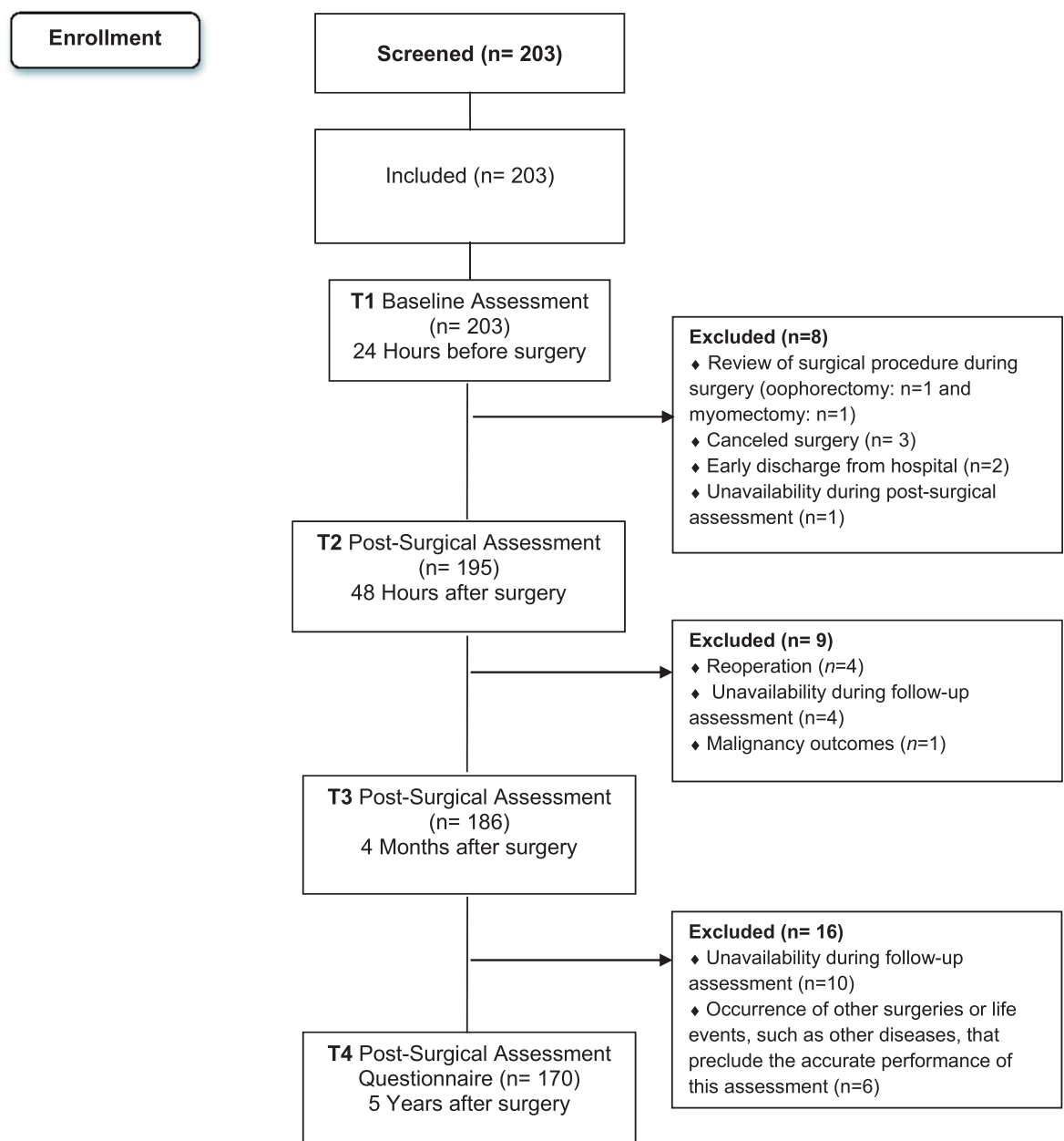

Figure 1. Flowchart of women undergoing hysterectomy.

influence my illness"); "treatment control" $(\alpha=0.76$; eg, "Surgery can control my illness"); "illness coherence" $(\alpha=$ 0.78; eg, "My illness is a mystery for me"); and "emotional representation" ( $\alpha=0.88$; eg, "When I think about my illness I get upset"). "Consequences" and "personal control" were not included because of their low score reliability in this sample $(\alpha$ $=0.56$ and $\alpha=0.53$, respectively). Each item is rated on a scale of 1 to 5 and to calculate each total subscale score, items are summed. Each subscale varies between 5 and 15, with high scores revealing less adaptive illness perceptions, with the exception of personal and treatment control subscales, which score inversely.

\subsubsection{The Coping Strategies Questionnaire-revised form}

Used to evaluate 6 pain coping strategies: "pain catastrophizing" (5 items; $\alpha=0.88$ ); "praying and hoping" (3 items; $\alpha=$ 0.88); "ignoring pain" (5 items; $\alpha=0.92)$; "distraction/diverting attention" (5 items; $\alpha=0.77$ ); "reinterpreting pain" ( 4 items; $\alpha$ $=0.74$ ); and "pain coping self-statements" ( 4 items; $\alpha=0.70$ ). During pilot testing, subjects were often confused by the usual 7-point Likert-type scale. ${ }^{87}$ Therefore, a 5-point rating scale was used $(1=$ never, 2 = almost never, $3=$ sometimes, $4=$ almost always, and 5 = always), which was shown to be more easily understood. The total subscale score was obtained by the sum of the item scores, with higher scores indicating greater use of the specific coping strategy.

\subsubsection{Life Orientation Test_revised}

Evaluated the personality trait optimism using 8 items. ${ }^{92}$ The total score ranges from 0 to $12(\alpha=0.95)$, with high values associated with more optimism.

\subsubsection{Postsurgical assessment-48 hours after surgery (T2)}

Forty-eight hours after surgery, women were again assessed in a face-to-face interview by the same psychologist who performed the baseline assessment in $\mathrm{T} 1$.

\subsubsection{Acute postsurgical pain measurement}

Worst and average levels of acute pain intensity, within the first 48 hours after surgery, were assessed using an 11-point NRS (from the BPI-SF described above). ${ }^{24}$

The PSP frequency assessment was performed using the frequency scale of the McGill Pain Questionnaire. ${ }^{66}$ Pain could be defined either as constant (continuous and steady), intermittent (periodic and rhythmic), or brief (momentary and transient).

\subsubsection{Postsurgical anxiety}

Postsurgical anxiety was evaluated again at this time point using the Hospital Anxiety and Depression Scale anxiety subscale ${ }^{110}$ already described. 


\subsubsection{Postsurgical assessment-4 months (T3) and 5 years after surgery (T4)}

Four months and 5 years after surgery, telephone calls were made to every participant to check for the presence of pain. The question was: "Do you still have any pain that you could link to surgery or that you could relate to the surgical procedure?" This is an adaptation of the BPI-SF first question on pain prospection. If women answered no, those women were classified as cases without pain. Contrarily, if women answered yes, they were considered pain cases. For those women reporting pain, additional measures were used, which focused on pain assessment.

\subsubsection{The Brief Pain Inventory-short form}

As described above. ${ }^{24}$

\subsubsection{Pain frequency description}

Pain frequency could be described as constant, daily, several times a week but not daily, several times a month but not weekly, during sexual intercourse, by touch or lifting weight.

\subsubsection{Neuropathic Pain Questionnaire (DN-4)}

Previous research described CPSP as a potential neuropathic pain. ${ }^{4,48,51}$ This instrument assesses pain characteristics/quality using 10 items. Seven of them refer to specific pain sensory descriptors, such as burning, pinpricking or numbness, and patients answer if their pain has those characteristics using a dichotomous response format (yes or no). The last 3 items result from the sensory examination of patients performed by a clinician. For the purpose of this study, only the first 7 items were included. ${ }^{5}$ Besides the information regarding pain quality, this questionnaire also provides information concerning the potential likelihood of neuropathic pain, corresponding to a cutoff of 3 (DN4 $\geq 3$ ).

\subsection{Surgical procedures, anesthetic, and analgesic techniques}

Clinical data related to the surgery, anesthesia and analgesia were collected from medical records.

Regarding surgical procedure, among the 170 women who underwent surgery, $122(71.8 \%)$ were submitted to total abdominal hysterectomy, 31 (18.2\%) to vaginal hysterectomy, $11(6.5 \%)$ to total laparoscopic hysterectomy, and $6(3.5 \%)$ to laparoscopically assisted vaginal hysterectomy. In abdominal hysterectomy ( $n=122)$, a Pfannenstiel incision was performed in 102 women (83.6\%), being established as the first choice, with a vertical infraumbilical incision being performed in 20 women (16.4\%), corresponding to cases wherein a previous vertical surgical scar was present.

Concomitant procedures, such as oophorectomy, ovarian cystectomy, salpingectomy, cystoscopy, or vaginal repair, were also performed in a few patients. Moreover, uterus weight and height were also recorded.

Concerning the type of anesthesia, 51 (30.0\%) patients had general anesthesia, 22 (12.9\%) had locoregional, and 97 (57.1\%) were submitted to combined anesthesia (general + locoregional). ASA score (physical status classification of the American Society of Anesthesiologists) was recorded, including cases of ASA grade I (49, 28.8\%), II (107, 62.9\%), and III (14, 8.2\%).

In what regards analgesic procedures, a postsurgical 48 hours standardized analgesia protocol was assigned to all patients. This protocol was established and supervised by the Acute Pain Service, before the transferring of patients to the infirmary.
Delivery of the analgesic protocol was either epidural or intravenous. The standardized epidural protocols could be: (1) a continuous epidural infusion delivered infusion balloon with ropivacaine $(0.1 \%)$ and fentanyl $(3 \mu \mathrm{g} / \mathrm{mL})$, administrated to 105 (61.8\%) women; or (2) administration of an epidural morphine bolus (2-3 mg, 12/12 h), assigned to $14(8.2 \%)$ women. The intravenous protocol consisted in a continuous intravenous infusion delivered infusion balloon of tramadol $(600 \mathrm{mg})$, metamizol (6 g), and metoclopramide (60 mg) and was used with 51 (30.0\%) patients.

Paracetamol (1 $\mathrm{g} \mathrm{6/6} \mathrm{h)} \mathrm{and} \mathrm{nonsteroidal} \mathrm{anti-inflammatory}$ drugs (ketorolac $30 \mathrm{mg}$ 12/12 h or parecoxib $40 \mathrm{mg}$ 12/12 h) were always included as coadjuvant analgesics. In addition, all analgesic regimens included prokinetic treatment that was standardized to metoclopramide (10 mg intravenously $8 / 8 \mathrm{~h}$ ). In cases of moderate or severe acute PSP levels (NRS > 3), rescue analgesia was prescribed beyond the standardized analgesic protocol. Because of the great variability in analgesics' protocol and dosages, no attempt was made to determine total equianalgesic medication dosages. Instead, it was recorded whether rescue analgesics were given to patients or not.

The use of psychotropic drugs (anxiolytics and antidepressants) during hospital stay was detailed from hospital records.

\subsection{Statistical analyses}

The primary outcome variable under study is PSP trajectory, corresponding to 3 possible PT groups: (1) No CPSP or pain trajectory 1 (PT1), comprised by women who did not report pain neither at 4 months nor at 5 years after surgery; (2) prolonged PSP or pain trajectory 2 (PT2), including women who complained of pain 4 months after surgery, but not 5 years after; and (3) CPSP or pain trajectory 3 (PT3), comprising those women who reported pain both at 4 months and 5 years after hysterectomy. These groups were defined taking into account pain report (yes [presence] or no [absence]) 4 months and 5 years after hysterectomy (T3 and T4).

To compare the 3 groups on the variables under analysis, chisquare or Fisher tests and one-way analysis of variance statistical tests were computed. For the latter, in the case of significant results $(P<0.05)$, Bonferroni post hoc tests were performed to further investigate between-group differences. These preliminary analyses were exploratory and were performed to determine the predictor variables to include in the subsequent regression analyses.

Finally, a set of predictive multinomial logistic regression models were conducted to investigate risk factors associated with PT group membership. The sociodemographic, clinical, and psychological variables selected for the regression analyses were those that distinguished PT groups in univariate analyses. Two structural models were developed: model 1 (M1), investigating the role of baseline presurgical variables (T1), allowing for the establishment of a presurgical risk profile; and model 2 (M2), aiming at further understanding the role of acute postsurgical factors, 48 hours after hysterectomy (T2). These basic structural models, include demographic (age) and clinical (pain-related [presurgical pain] and surgery-related [type of hysterectomy]) variables that significantly distinguished the groups in univariate analyses, to control these effects in group comparisons.

Considering the role of presurgical baseline predictors, and due to shared variance among the 5 psychological predictors (and consequent multicollinearity), which emerged as significant in the distinction of the 3 groups, 3 different submodels were considered. The first model (M1A) focused on emotional variables 
(presurgical anxiety and fear), the second (M1B) centered on illness perceptions (emotional representation of the disease underlying surgery), and the third model (M1C) tested the role of pain coping strategies (pain catastrophizing).

Regarding acute postsurgical factors, 2 models were computed, both controlling for the same abovementioned demographic and clinical variables that were controlled for in presurgical models. Beyond these covariates, the first model (M2A) tested for the specific predictive role of acute PSP intensity and frequency. The second postsurgical model (M2B) was akin to the first, although it had the addition of postsurgical anxiety.

Data were analyzed using the IBM SPSS Statistics version 24.0. Internal consistency of responses to the questionnaires was assessed using Cronbach alpha. ${ }^{27}$ Results were considered significant for $P$ values $<0.05$. Effect size (ES) measures were interpreted considering the Cohen ${ }^{25}$ rule of thumb for eta squared $\left(\eta^{2}\right)$ : small ES $=0.02$; medium $E S=0.13$; large $E S=0.26$; and Rea and Parker ${ }^{84}$ for Cramer phi $(\varphi)$ or Cramer $\bigvee$ for nominal data: negligible association: 0 and under 0.10; weak association: 0.10 and under 0.20; moderate association: 0.20 and under 0.40; relatively strong association: 0.40 and under 0.60; strong association: 0.60 and under 0.80 ; and very strong association: 0.80 and under 1.00 .

\section{Results}

\subsection{Pain trajectory according to pain report 4 months (T3) and 5 years (T4) after hysterectomy}

Table 1 presents the number of women within each PT group, according to the course of pain up to 5 years after surgery.

Considering PT, 88 (51.8\%, PT1) women did not complain of pain at 4 months or 5 years after surgery. This was the largest group and was called "No Chronic Post-Surgical Pain." This PT is a typical, expected, and desirable one in terms of healing and normal recovery. The second group was comprised of 53 (31.2\%, PT2) women who complained of pain 4 months postsurgery, but at 5 years after surgery did not experience any related pain. This group was labelled "Prolonged Post-Surgical Pain." The third PT group (PT3) included 29 (17.1\%) women who reported pain both at 4 months and 5 years after hysterectomy. As their pain persisted over time, this group was labelled "Chronic PostSurgical Pain." All patients reporting pain at the 5-year follow-up also had reported pain at 4 months postsurgery. Patients not reporting pain at the 4-month follow-up also did not report pain 5 years later. Thus, there were no cases of "new pain" with a later onset beyond the 4-month follow-up time point.

\subsection{Differences among pain trajectory groups on sociodemographic, clinical, and psychological variables}

Table 2 shows that before surgery, PT groups differed in age $\left(P=0.013 ; \eta^{2}=0.051\right.$, small ES), with PT1 being older than
PT2 $(P=0.003)$. Concerning clinical measures, the groups were similar in terms of surgical disease onset, body mass index, previous surgical procedures, or presurgical psychotropic use. Regarding presurgical pain, the 3 groups of women differed significantly on presurgical pain related to the condition underlying surgery $(P=0.032 ; \varphi=0.201$, moderate association), but not on previous chronic pain because of other causes (Table 2). Among the 3 empirically derived PT groups, and regarding psychological variables measured before surgery, there were significant differences in anxiety $(P<$ $0.001 ; \eta^{2}=0.094$, small ES), surgical fear related to long-term consequences of surgery ( $P=0.022 ; \eta^{2}=0.045$, small ES), emotional representation of surgical disease $\left(P=0.001 ; \eta^{2}=\right.$ 0.081 , small ES), and pain catastrophizing $\left(P<0.001 ; \eta^{2}=\right.$ 0.090, small ES). For all these variables, higher values were associated with chronic pain group membership in PT2 or PT3.

In terms of surgery, the PT groups could be distinguished based on the type of surgical approach $(P=0.007 ; \varphi=0.242$, moderate association) and among those undergoing abdominal hysterectomy, there were also differences across groups on the type of abdominal incision $(P=0.003 ; \varphi=0.260$, moderate association). The PT2 and PT3 groups had more women undergoing abdominal hysterectomy and a Pfannenstiel incision.

In the acute postsurgical period, 48 hours after surgery, the 3 PT groups of women showed differences in acute pain report, regarding worst $\left(P<0.001 ; \eta^{2}=0.096\right.$, small ES) and average $\left(P=0.001 ; \eta^{2}=0.086\right.$, small ES) pain intensity, pain frequency ( $P=0.001 ; \varphi=0.286$, moderate association), and postsurgical anxiety $\left(P<0.001 ; \eta^{2}=0.109\right.$, small ES). These differences showed that the PT2 and PT3 groups scored more negatively on these variables.

\subsection{Pain incidence, characteristics, and perceived impact 5 years after hysterectomy}

Among the 170 women who completed the 4 assessments, 29 (17.1\%) revealed an unfavorable PT after hysterectomy, reporting pain both at 4 months and 5 years after surgery, therefore being considered CPSP cases (Table 3). From these 29 cases of CPSP, Table 3 highlights that in terms of pain frequency, most women (44.8\%) reported pain several times a week, with $24.2 \%$ of women perceiving pain on a daily basis and $20.7 \%$ complaining of pain only during sexual intercourse or by touch. In addition, the mean of worst pain intensity was $3.17(\mathrm{SD}=1.61)$ and the mean average level of pain intensity was $1.89(\mathrm{SD}=0.88)$, on the 0 to 10 NRS. It was also shown that $34.5 \%$ of CPSP women rated their worst pain intensity above 3 (NRS > 3), indicating moderate and/ or severe pain levels. Table 3 also reveals that the pain sensory characteristics more often described by patients with CPSP were feeling of pins and needles (51.7\%) and numbness (31.0\%). In terms of a potential neuropathic pain component of CPSP, 5 (17.2\%) women presented a DN4 $\geq 3$ value. Pain interference

Table 1

Pain trajectory groups according to pain report at 4 months (T3) and 5 years (T4) after hysterectomy.

\begin{tabular}{|c|c|c|c|c|}
\hline \multirow[t]{2}{*}{ Pain trajectories } & \multicolumn{2}{|c|}{ Chronic pain report $(\mathrm{N}=170)$} & \multirow[t]{2}{*}{ n (\%) } & \multirow[t]{2}{*}{ Group designation } \\
\hline & T3 (4 mo after surgery) & T4 (5 y after surgery) & & \\
\hline Pain trajectory 1 & No & No & $88(51.8)$ & No chronic postsurgical pain \\
\hline Pain trajectory 2 & Yes & No & $53(31.2)$ & Prolonged postsurgical pain \\
\hline Pain trajectory 3 & Yes & Yes & $29(17.1)$ & Chronic postsurgical pain \\
\hline
\end{tabular}


Table 2

Differences between pain trajectory groups on sociodemographic, clinical, and psychological variables measured 24 hours before (T1) and 48 hours after hysterectomy (T2).

\begin{tabular}{|c|c|c|c|c|c|c|}
\hline Measures & PT1 (n = 88) & PT2 (n = 53) & PT3 (n = 29) & $\mathrm{F} / \mathrm{\chi}^{2}$ & $P$ & $\eta^{2} / \varphi$ \\
\hline \multicolumn{7}{|l|}{ Presurgical data 24 h before surgery-T1 } \\
\hline Sociodemographic: Age (y [SD]) & $52.8(10.2)^{\mathrm{a}}$ & $48.7(6.43)^{b}$ & $48.7(8.19)^{\mathrm{ab}}$ & 4.446 & 0.013 & 0.051 \\
\hline \multicolumn{7}{|l|}{ Presurgical clinical variables } \\
\hline Disease onset (mo) & $39.2(48.6)$ & $36.3(46.0)$ & $44.9(71.4)$ & 0.250 & 0.779 & 0.003 \\
\hline $\mathrm{BMI}\left(\mathrm{kg} / \mathrm{m}^{2}\right)$ & $28.8(4.26)$ & $29.0(4.78)$ & $27.9(4.15)$ & 0.585 & 0.558 & 0.007 \\
\hline Previous surgeries (yes) & $62(70.5 \%)$ & $37(69.8 \%)$ & $21(72.4 \%)$ & 0.063 & 0.969 & 0.019 \\
\hline Psychotropic use* (yes) & $28(33.7 \%)$ & $19(37.3 \%)$ & $14(48.3 \%)$ & 1.941 & 0.379 & 0.109 \\
\hline Presurgical pain (yes) & $44(50.0 \%)$ & $36(67.9 \%)$ & $21(72.4 \%)$ & 6.858 & 0.032 & 0.201 \\
\hline Previous pain due to other causest (yes) & $49(55.7 \%)$ & $37(69.8 \%)$ & $21(72.4 \%)$ & 4.176 & 0.124 & 0.157 \\
\hline \multicolumn{7}{|l|}{ Psychological variables (range) } \\
\hline HADS: anxiety (0-21) & $6.02(3.84)^{b}$ & $7.87(3.96)^{\mathrm{a}}$ & $9.38(4.81)^{\mathrm{a}}$ & 8.615 & $<0.001$ & 0.094 \\
\hline HADS: depression (0-21) & $1.89(2.48)$ & $2.68(3.71)$ & $2.69(2.93)$ & 1.509 & 0.224 & 0.018 \\
\hline SFQ: immediate consequences (0-40) & $9.02(8.55)$ & $10.8(7.26)$ & $13.3(10.1)$ & 2.892 & 0.058 & 0.033 \\
\hline SFQ: long-term consequences (0-40) & $4.61(6.78)^{\mathrm{b}}$ & $4.66(5.33)^{b}$ & $8.41(8.12)^{\mathrm{a}}$ & 3.929 & 0.022 & 0.045 \\
\hline IPQ-R: timeline acute/chronic (3-15) & $6.11(1.39)$ & $5.98(1.01)$ & $6.52(1.53)$ & 1.607 & 0.204 & 0.019 \\
\hline IPQ-R: timeline cyclical (3-15) & $6.94(3.09)$ & $7.85(3.20)$ & $8.28(3.36)$ & 2.530 & 0.083 & 0.029 \\
\hline IPQ-R: treatment control (3-15) & $12.2(0.98)$ & $12.2(0.77)$ & $12.1(0.75)$ & 0.231 & 0.794 & 0.003 \\
\hline IPQ-R: illness coherence (3-15) & $9.27(2.76)$ & $9.36(2.60)$ & $9.90(2.58)$ & 0.604 & 0.548 & 0.007 \\
\hline IPQ-R: emotional representation (3-15) & $7.60(2.89)^{b}$ & $9.04(2.90)^{\mathrm{a}}$ & $9.62(2.68)^{\mathrm{a}}$ & 7.383 & 0.001 & 0.081 \\
\hline CSQ-R: pain catastrophizing (6-30) & $9.25(4.28)^{\mathrm{b}}$ & $11.3(5.81)^{\mathrm{ab}}$ & $13.6(6.50)^{\mathrm{a}}$ & 8.232 & $<0.001$ & 0.090 \\
\hline CSQ-R: praying/hoping (3-15) & $9.57(4.22)$ & $10.0(4.04)$ & 10.5 (3.67) & 0.560 & 0.573 & 0.007 \\
\hline CSQ-R: ignoring (5-25) & $12.4(5.81)$ & $11.9(6.18)$ & $11.0(6.12)$ & 0.568 & 0.568 & 0.007 \\
\hline CSQ-R: diverting attention (5-25) & $9.70(4.70)$ & $10.2(4.54)$ & $9.86(4.76)$ & 0.213 & 0.808 & 0.003 \\
\hline CSQ-R: reinterpreting pain (4-20) & $6.71(3.23)$ & $7.00(3.52)$ & $6.96(3.88)$ & 0.133 & 0.876 & 0.002 \\
\hline CSQ-R: pain coping self-stat (4-20) & $16.0(3.61)$ & $15.9(3.53)$ & $14.9(3.57)$ & 1.092 & 0.338 & 0.014 \\
\hline LOT-R: optimism (0-12) & $7.30(3.48)$ & $6.63(3.01)$ & $7.14(3.25)$ & 0.663 & 0.517 & 0.008 \\
\hline \multicolumn{7}{|l|}{ Postsurgical variables-T2 } \\
\hline Type hystł: open abdominal & $54(61.4 \%)$ & $43(81.1 \%)$ & $25(86.2 \%)$ & 9.978 & 0.007 & 0.242 \\
\hline Abdom. Incis.§: Pfannenstiel & $42(47.7 \%)$ & $39(73.6 \%)$ & $21(72.4 \%)$ & 11.460 & 0.003 & 0.260 \\
\hline Uterus weight (g) & $219(220)$ & $217(207)$ & $205(213)$ & 0.045 & 0.956 & 0.001 \\
\hline Uterus height (cm) & $9.61(2.65)$ & $9.30(2.70)$ & $9.49(2.62)$ & 0.228 & 0.797 & 0.003 \\
\hline Worst pain intensityll & $4.40(2.85)^{b}$ & $5.70(2.79)^{\mathrm{a}}$ & $6.86(3.03)^{\mathrm{a}}$ & 8.853 & $<0.001$ & 0.096 \\
\hline Average pain intensityll & $2.59(1.61)^{b}$ & $3.55(1.58)^{\mathrm{a}}$ & $3.55(1.43)^{\mathrm{a}}$ & 7.901 & 0.001 & 0.086 \\
\hline Pain frequency $\Phi:$ constant & $17(19.3 \%)$ & $18(34.0 \%)$ & $16(55.2 \%)$ & 13.928 & 0.001 & 0.286 \\
\hline HADS: anxiety & $2.05(2.50)^{b}$ & $3.23(3.83)^{b}$ & $5.21(4.36)^{\mathrm{a}}$ & 10.18 & $<0.001$ & 0.109 \\
\hline Psychotropic use ${ }^{\star}$ (yes) & $33(37.5 \%)$ & $21(39.6 \%)$ & $14(50 \%)$ & 1.392 & 0.498 & 0.091 \\
\hline Length of stay (d) & $3.10(0.94)$ & $3.00(0.56)$ & $3.00(0.48)$ & 0.373 & 0.689 & 0.005 \\
\hline
\end{tabular}

Continuous variables are presented as mean (SD); categorical variables are presented as $n(\%)$.

Different letters represent $P$ values $<0.05$ in analysis of variance post hoc tests; for example, in age groups comparisons, PT1 (represented with a) is significantly different from PT2 (represented with b) and PT3 does not significantly differ from PT1 and PT2 (represented with ab).

* Psychotropic use: consumption/intake of anxiolytics and antidepressants.

† Other previous chronic pain states not related to the cause of surgery.

$\ddagger$ Type of hysterectomy: $n(\%)$ of open abdominal hysterectomies vs abdominal laparoscopic, vaginal, and vaginal-assisted laparoscopic.

$\S$ Abdominal incision: $\mathrm{n}(\%)$ of Pfannenstiel incisions vs infraumbilical vertical incision and laparoscopies.

$\| \mathrm{NRS}=$ numerical rating scale 0 to 10 from Brief Pain Inventory (BPI).

- Pain frequency: constant pain vs intermittent or brief pain, assessed through frequency subscale of McGill Pain Questionnaire.

BMI, body mass index; CSQ-R, Coping Strategies Questionnaire-Revised; HADS, Hospital Anxiety and Depression Scale; IPQ-R, Illness Perception Questionnaire-Revised; LOT-R, Life Orientation Test-revised; PT, pain trajectory; SFQ, Surgical Fear Questionnaire.

was reported in all domains, the most common being mood (57.1\%), enjoyment of life (50.0\%), general activity (42.9\%), normal work (42.9\%), and walking ability (28.6\%). Nevertheless, mean values of pain interference were in the low-to-medium range.

\subsection{Predictors of postsurgical pain trajectory}

To identify the presurgical predictors of an unfavorable PT, 3 multinomial regression models were computed (Table 4), controlling for the demographic and clinical variables that distinguished the 3 groups: age, previous surgical pain, and type of hysterectomy. The first model (M1A) further tested for the influence of psychological factors related to emotional distress, namely presurgical anxiety and surgical fear related to the longterm consequences of surgery. Table 4 shows that presurgical anxiety is a predictor of PT, being a determinant of group membership, with higher anxiety scores being associated with both the "Prolonged PSP group" (PT2; odds ratio [OR] = 1.131, $P$ $=0.015$; for each unit increased in presurgical anxiety, the odds of being in the PT2 group is 1.131 times higher than those of being in the PT1 group) and the "CPSP group" (PT3; OR $=1.175, P=$ 0.009), when compared with the "No CPSP group" (PT1). All other variables were not significant. Table 4 also reveals results on the influence of psychological factors associated with illness perceptions (M1B) and pain coping strategies (M1C). Within 
Table 3

Incidence, characteristics, and impact of pain 5 years after hysterectomy ( $\mathbf{N}=29)$.

\begin{tabular}{lrll}
\hline Pain $\mathbf{5}$ y after hysterectomy-T4 & N (\%) & M (SD) & Min-max \\
\hline Pain report-CPSP* & $29(17.1)$ & & \\
\hline Frequency & & & \\
$\quad$ Daily & $7(24.2)$ & & \\
$\quad$ Several times a week but not daily & $13(44.8)$ & & \\
$\quad$ Several times a month but not weekly & $3(10.3)$ & & \\
$\quad$ During sexual intercourse/by touch & $6(20.7)$ & & \\
\hline Intensity† (NRS 0-10) & & $3.17(1.61)$ & $1-7$ \\
$\quad$ Worst level & $10(34.5)$ & & \\
$\quad$ NRS $>3$ & $1.89(0.88)$ & $1-4$ \\
$\quad$ Average level & $1(3.4)$ & & \\
$\quad$ NRS $>3$ & & &
\end{tabular}

DN-4ł (could report 1 or more)

$\begin{array}{lr}\text { Pins and needles } & 15(51.7) \\ \text { Numbness } & 9(31.0) \\ \text { Itching } & 6(20.7) \\ \text { Tingling } & 3(10.3) \\ \text { Burning } & 2(6.9) \\ \text { Painful cold } & 1(3.4) \\ \text { Electric shocks } & 1(3.4) \\ \text { DN4 }<3 & 23(82.8) \\ \text { DN4 } \geq 3 & 5(17.2)\end{array}$

Pain interference§ (NRS 0-10) General activity Mood Walking ability Normal work Relations with other people Sleep Enjoyment of life

$\begin{array}{ccc}12(42.9) & 1.07(1.41) & 0-4 \\ 16(57.1) & 1.64(1.83) & 0-5 \\ 8(28.6) & 0.57(1.03) & 0-3 \\ 12(42.9) & 1.14(1.56) & 0-5 \\ 5(17.8) & 0.32(0.77) & 0-3 \\ 2(7.2) & 1.79(0.67) & 0-3 \\ 14(50) & 1.32(1.68) & 0-5\end{array}$

Continuous variables are presented as mean (SD); categorical variables are presented as $\mathrm{n}(\%)$.

* Women reporting CPSP—chronic postsurgical pain 5 years after hysterectomy.

† NRS: numerical rating scale (0-10).

$\ddagger$ DN-4: Neuropathic Pain Questionnaire.

§ Items from BPI-SF: Brief Pain Inventory—short form.

M1B, emotional representation of the condition underlying surgery emerged as the only factor significantly associated with group membership, with high scores predicting PT2 (OR = 1.165, $P=0.018)$ and PT3 $(\mathrm{OR}=1.246, P=0.005)$ membership. Within $\mathrm{M} 1 \mathrm{C}$, pain catastrophizing was the only variable yielding a significant association with either membership in PT2 (OR $=1.079, P=0.043)$ or PT3 (OR $=1.143, P=0.001)$ groups. Concerning factors that could distinguish PT2 from PT3 membership, Table 4 indicates that the psychological construct fear of long-term consequences of surgery was the only significant predictor $(\mathrm{OR}=1.078, P=0.049)$.

To further explore the role of acute postsurgical variables in the development of an unfavorable PT, over and above demographic and clinical factors, 2 subsequent models were tested (Table 5). The first model (M2A) revealed that acute PSP intensity and frequency determined inclusion of women in PT3 $(\mathrm{OR}=1.211, P=0.033$ and $\mathrm{OR}=3.000, P=0.029$, respectively). The second postsurgical model (model 2B) added postsurgical anxiety to the variables of the previous model to test its predictive role. Subsequently, both acute PSP intensity and frequency ceased to be significant and postsurgical anxiety became the only variable predicting PT3 (CPSP) $(\mathrm{OR}=1.182, P=0.026)$. Age, previous pain, and type of hysterectomy did not contribute to the prediction of an unfavorable PT in any of the models (Tables 4 and 5).

\section{Discussion}

This prospective cohort study followed women up to 5 years after hysterectomy and found that presurgical anxiety, emotional illness representations, and pain catastrophizing were risk factors for PSP. Higher postsurgical anxiety, acute pain intensity, and frequency increased the likelihood of a worst PT. These results are novel because there are no prospective studies on CPSP after hysterectomy with such a long follow-up and that consider the trajectory of pain over 2 long-term follow-up times ( 4 months and 5 years posthysterectomy). This study also adds to previous findings regarding pain at 48 hours $^{75,76}$ and 4 months $^{79}$ postsurgery.

\subsection{Chronic postsurgical pain prevalence after hysterectomy}

Almost half of women (48.2\%) reported pain 4 months after hysterectomy. Five years later, $17.1 \%$ still complained of pain. Within hysterectomy studies, the longest follow-up period that we are aware of (2 years) revealed a CPSP prevalence of $24.1 \%$ and $16 \%$ in abdominal and vaginal hysterectomy, respectively. ${ }^{67}$ With a 1-year follow-up, Theunissen et al. ${ }^{100}$ reported a prevalence of $9 \%$, although the criteria underlying CPSP definition was a cutoff level of 3 (NRS > 3), indicative of moderate or severe pain intensity. A retrospective study ${ }^{7}$ reported $31.9 \%$ prevalence and in a prospective study, ${ }^{6} 4$ months postsurgery and using a different CPSP definition (pain with impact on daily living), the prevalence rate was $16.7 \%$. Two other studies described a prevalence of $14 \%{ }^{103}$ and $26 \%,{ }^{80} 6$ months posthysterectomy.

These figures clearly reveal large discrepancies in CPSP report, most likely due to the diverse range of follow-up times and CPSP definitions. ${ }^{48,69}$ The relatively higher prevalence of CPSP found in our study may be due to not having included only those women reporting moderate to severe pain (NRS > 3). On the other hand, our findings show that pain only interfered with the lives of some women and that interference was in the low-to-medium range.

\subsection{Predictors of chronic postsurgical pain after hysterectomy}

\subsubsection{Demographic and clinical factors}

In contrast to earlier findings, $, 6,7,67,79,97,100,103$ age and presurgical pain did not predict CPSP. Studies that found such relationships had shorter follow-ups, from 4 months to 2 years. Consistent with previous results, $3,6,7,97,100,103$ hysterectomy type did not predict CPSP albeit this does not support our earlier findings, ${ }^{79}$ nor Montes et al. ${ }^{67}$ conclusions. These results were unexpected and suggest that the strength of association of demographic and clinical factors probably diminishes with time, as psychological factors play an increasingly greater role. A similar conclusion was drawn previously in a postmastectomy study with a 3-year follow-up. ${ }^{2}$

Acute PSP intensity is a well-established key predictive factor, ${ }^{13,14,52,55,61,62}$ including in hysterectomy CPSP studies. $6,80,97,100,103$ Present results support this and further add PSP frequency, as previously found in this sample. ${ }^{76}$ To the best of our knowledge, only Fletcher et al. ${ }^{35}$ found pain frequency to be a CPSP predictor. These results highlight pain frequency as a new potential target in CPSP prevention. When adding postsurgical anxiety to the equation, both acute pain predictors ceased to be significant, which indicates both their shared variance and the unique contribution of postsurgical anxiety in the prediction of CPSP. 
Table 4

Multinomial logistic regression model relating pain trajectory membership with presurgical variables measured 24 hours before hysterectomy.

\begin{tabular}{|c|c|c|c|c|c|c|c|c|}
\hline \multirow[t]{2}{*}{ Pain trajectory comparison } & \multirow[t]{2}{*}{ Presurgical models } & \multirow[t]{2}{*}{ Nagelkerke $R^{2}$} & \multirow[t]{2}{*}{ B } & \multirow[t]{2}{*}{ SE } & \multirow[t]{2}{*}{ Wald } & \multirow[t]{2}{*}{ OR } & \multicolumn{2}{|c|}{$95 \% \mathrm{Cl}$} \\
\hline & & & & & & & $\overline{\mathrm{LL}}$ & UL \\
\hline & Model 1A-emotional distress & 0.206 & & & & & & \\
\hline \multirow[t]{5}{*}{ PT1 vs PT2 } & Age† & & -0.036 & 0.024 & 2.334 & 0.964 & 0.920 & 1.010 \\
\hline & Presurgical pain $\ddagger$ & & 0.231 & 0.416 & 0.309 & 1.260 & 0.557 & 2.851 \\
\hline & Type of hysterectomy§ & & 0.750 & 0.447 & 2.820 & 2.118 & 0.882 & 5.084 \\
\hline & Presurgical anxiety\|l & & 0.123 & 0.051 & $5.924^{*}$ & 1.131 & 1.024 & 1.249 \\
\hline & Presurgical fear $\Phi$ & & -0.044 & 0.034 & 1.677 & 0.957 & 0.895 & 1.023 \\
\hline \multirow[t]{5}{*}{ PT1 vs PT3 } & Age $†$ & & -0.013 & 0.032 & 0.157 & 0.987 & 0.927 & 1.052 \\
\hline & Presurgical pain $\ddagger$ & & 0.706 & 0.534 & 1.752 & 2.026 & 0.712 & 5.767 \\
\hline & Type of hysterectomy§ & & 1.010 & 0.621 & 2.650 & 2.747 & 0.814 & 9.273 \\
\hline & Presurgical anxiety\|l & & 0.161 & 0.062 & $6.799^{\star \star}$ & 1.175 & 1.041 & 1.326 \\
\hline & Presurgical fear $\Phi$ & & 0.031 & 0.035 & 0.802 & 1.032 & 0.964 & 1.104 \\
\hline \multirow[t]{6}{*}{ PT2 vs PT3 } & Age† & & 0.024 & 0.034 & 0.471 & 1.024 & 0.957 & 1.095 \\
\hline & Presurgical pain $\ddagger$ & & 0.475 & 0.557 & 0.726 & 1.608 & 0.540 & 4.790 \\
\hline & Type of hysterectomy§ & & 0.260 & 0.669 & 0.151 & 1.297 & 0.350 & 4.809 \\
\hline & Presurgical anxiety\|l & & 0.038 & 0.062 & 0.376 & 1.039 & 0.920 & 1.173 \\
\hline & Presurgical fear $\Phi$ & & 0.075 & 0.038 & $3.880^{*}$ & 1.078 & 1.000 & 1.162 \\
\hline & Model 1Bł‡一illness perceptions & 0.171 & & & & & & \\
\hline \multirow[t]{4}{*}{ PT1 vs PT2 } & Age† & & -0.030 & 0.023 & 1.697 & 0.970 & 0.928 & 1.015 \\
\hline & Presurgical pain $\ddagger$ & & 0.263 & 0.405 & 0.423 & 1.301 & 0.588 & 2.878 \\
\hline & Type of hysterectomy§ & & 0.793 & 0.445 & 3.168 & 2.209 & 0.923 & 5.290 \\
\hline & Emotional representation\# & & 0.152 & 0.064 & $5.586^{*}$ & 1.165 & 1.026 & 1.321 \\
\hline \multirow[t]{5}{*}{ PT1 vs PT3 } & Age $†$ & & -0.018 & 0.030 & 0.378 & 0.982 & 0.926 & 1.041 \\
\hline & Presurgical pain $\neq$ & & 0.430 & 0.515 & 0.698 & 1.538 & 0.560 & 4.219 \\
\hline & Type of hysterectomy§ & & 1.166 & 0.621 & 3.530 & 3.209 & 0.951 & 10.829 \\
\hline & Emotional representation\# & & 0.220 & 0.079 & $7.821^{\star \star}$ & 1.246 & 1.068 & 1.455 \\
\hline & Model 1C $\neq \ddagger$-pain coping strategies & 0.178 & & & & & & \\
\hline \multirow[t]{4}{*}{ PT1 vs PT2 } & Age† & & -0.037 & 0.023 & 2.611 & 0.963 & 0.921 & 1.008 \\
\hline & Presurgical pain $\ddagger$ & & 0.373 & 0.402 & 0.862 & 1.452 & 0.661 & 3.190 \\
\hline & Type of hysterectomy§ & & 0.609 & 0.045 & 1.878 & 1.839 & 0.769 & 4.395 \\
\hline & Pain catastrophizing†† & & 0.076 & 0.038 & $4.086^{*}$ & 1.079 & 1.002 & 1.162 \\
\hline \multirow[t]{4}{*}{ PT1 vs PT3 } & Age† & & -0.034 & 0.031 & 1.172 & 0.967 & 0.910 & 1.028 \\
\hline & Presurgical pain $\ddagger$ & & 0.577 & 0.520 & 1.231 & 1.781 & 0.642 & 4.940 \\
\hline & Type of hysterectomy§ & & 0.798 & 0.623 & 1.641 & 2.221 & 0.655 & 7.532 \\
\hline & Pain catastrophizing†† & & 0.134 & 0.042 & $10.170^{\star \star \star}$ & 1.143 & 1.053 & 1.241 \\
\hline
\end{tabular}

${ }^{\star} P<0.05 ;{ }^{* \star} P<0.01 ;$ and ${ }^{\star \star \star} P<0.001$.

PT1-Reference Group (In MODEL 1A PT2 vs PT3, PT2 is the reference group).

$\dagger$ Continuous variable, in years.

$\ddagger$ Dichotomous variable: $0=$ no and $1=$ yes.

$\S$ Dichotomous variable: $0=$ abdominal laparoscopic, vaginal, and vaginal-assisted laparoscopic and $1=$ open abdominal hysterectomies.

II Continuous variable, HADS-A: Hospital Anxiety and Depression Scale-anxiety subscale.

I Continuous variable, Surgical Fear Questionnaire-fear of immediate consequences of surgery subscale.

\# Continuous variable, IPQ-R: IIIness Perception Questionnaire-Revised—all subscales.

t† Continuous variable, CSQ-R: Coping Strategies Questionnaire-Revised-all subscales.

㧊 For models $1 \mathrm{~B}$ and $1 \mathrm{C}$, no significant predictors were found in the comparison of PT2 and PT3.

$\mathrm{Cl}$, confidence interval; LL, lower limit; OR, odds ratio; PT1, pain trajectory group 1 (no chronic postsurgical pain); PT2, pain trajectory group 2 (prolonged postsurgical pain); PT3, pain trajectory group 3 (chronic postsurgica pain); UL, upper limit.

It is noteworthy that the acute postsurgical variables only predicted pain 5 years later. This suggests that to prevent longterm posthysterectomy pain, women should be screened and targeted throughout the perioperative period, and not only before surgery. Baseline assessments are useful for medium- and longterm pain prediction, whereas acute postsurgical variables seem to better predict long-term chronic pain.

\subsubsection{The key role of psychological predictors}

Presurgical anxiety and pain catastrophizing were predictive of an unfavorable PSP trajectory and CPSP, which corroborates previous findings. ${ }^{16,44,99}$ Presurgical anxiety, already shown to be a predictor of CPSP 4 and 6 months s9, $^{703}$ posthysterectomy, is also predictive of CPSP 5 years later. Furthermore, pain catastrophizing, a well-established CPSP psychological risk factor, $2,15,50,56,83$ emerges as a predictor for the first time in our hysterectomy studies. ${ }^{79}$

Our results also confirm the important role of emotional representation of the surgical disease and of postsurgical anxiety. ${ }^{79}$ Other studies have demonstrated the impact of cognitive and affective responses triggered by illness on health outcomes, ${ }^{57,91}$ although the investigation of their predictive role on PSP has been scarce. ${ }^{77,79}$ Postsurgical anxiety is such a response, present findings highlighting the need to address anxiety throughout the perioperative period, and not only before surgery. 
Table 5

Multinomial logistic regression model relating pain trajectory membership with postsurgical variables measured 48 hours after hysterectomy.

\begin{tabular}{|c|c|c|c|c|c|c|c|c|}
\hline \multirow[t]{2}{*}{ Pain trajectory comparison } & \multirow[t]{2}{*}{ Postsurgical models } & \multirow[t]{2}{*}{ Nagelkerke $R^{2}$} & \multirow[t]{2}{*}{ B } & \multirow[t]{2}{*}{ SE } & \multirow[t]{2}{*}{ Wald } & \multirow[t]{2}{*}{ OR } & \multicolumn{2}{|c|}{$95 \% \mathrm{Cl}$} \\
\hline & & & & & & & $\overline{\mathrm{LL}}$ & UL \\
\hline & Model 2A††-acute pain & 0.192 & & & & & & \\
\hline \multirow[t]{5}{*}{ PT1 vs PT2 } & Age & & -0.031 & 0.023 & 1.774 & 0.970 & 0.927 & 1.015 \\
\hline & Presurgical pain $\ddagger$ & & 0.289 & 0.404 & 0.511 & 1.335 & 0.605 & 2.946 \\
\hline & Type of hysterectomy§ & & 0.640 & 0.443 & 2.083 & 1.896 & 0.795 & 4.552 \\
\hline & Acute postsurgical pain intensityll & & 0.067 & 0.069 & 0.957 & 1.070 & 0.935 & 1.224 \\
\hline & Acute postsurgical pain frequency & & 0.429 & 0.431 & 0.992 & 1.535 & 0.660 & 3.571 \\
\hline \multirow[t]{6}{*}{ PT1 vs PT3 } & Age & & -0.011 & 0.032 & 0.114 & 0.989 & 0.929 & 1.053 \\
\hline & Presurgical pain $\ddagger$ & & 0.388 & 0.527 & 0.543 & 1.474 & 0.525 & 4.139 \\
\hline & Type of Hysterectomy§ & & 0.765 & 0.634 & 1.457 & 2.149 & 0.620 & 7.441 \\
\hline & Acute postsurgical pain intensityll & & 0.191 & 0.090 & $4.525^{\star}$ & 1.211 & 1.015 & 1.444 \\
\hline & Acute postsurgical pain frequency & & 1.099 & 0.505 & $4.741^{*}$ & 3.000 & 1.116 & 8.065 \\
\hline & Model 2B十†-Acute pain + anxiety & 0.222 & & & & & & \\
\hline \multirow[t]{6}{*}{ PT1 vs PT2 } & Age† & & -0.031 & 0.023 & 1.743 & 0.970 & 0.926 & 1.015 \\
\hline & Presurgical pain $\ddagger$ & & 0.360 & 0.410 & 0.771 & 1.433 & 0.642 & 3.201 \\
\hline & Type of hysterectomy§ & & 0.637 & 0.446 & 2.041 & 1.890 & 0.789 & 4.527 \\
\hline & Acute postsurgical pain intensity\|l & & 0.031 & 0.074 & 0.172 & 1.031 & 0.892 & 1.192 \\
\hline & Acute postsurgical pain frequency & & 0.306 & 0.445 & 0.474 & 1.358 & 0.568 & 3.250 \\
\hline & Postsurgical anxiety\# & & 0.093 & 0.068 & 1.863 & 1.098 & 0.960 & 1.255 \\
\hline \multirow[t]{6}{*}{ PT1 vs PT3 } & Age† & & -0.016 & 0.033 & 0.234 & 0.984 & 0.923 & 1.050 \\
\hline & Presurgical pain $\ddagger$ & & 0.430 & 0.536 & 0.643 & 1.537 & 0.538 & 4.391 \\
\hline & Type of hysterectomy§ & & 0.715 & 0.646 & 1.224 & 2.044 & 0.576 & 7.255 \\
\hline & Acute postsurgical pain intensity\|l & & 0.123 & 0.097 & 1.620 & 1.131 & 0.936 & 1.367 \\
\hline & Acute postsurgical pain frequency & & 0.786 & 0.536 & 2.152 & 2.195 & 0.768 & 6.277 \\
\hline & Postsurgical anxiety\# & & 0.167 & 0.075 & $4.945^{\star}$ & 1.182 & 1.020 & 1.370 \\
\hline
\end{tabular}

${ }^{\star} P<0.05 ;{ }^{* \star} P<0.01 ;$ and ${ }^{\star \star \star} P<0.001$.

PT1-Reference Group.

† Continuous variable, in years.

$\ddagger$ Dichotomous variable: $0=$ no and $1=$ yes.

$\S$ Dichotomous variable: $0=$ abdominal laparoscopic, vaginal, and vaginal-assisted laparoscopic and $1=$ open abdominal hysterectomies.

\| Continuous variable, NRS 0 to 10 from BPI-SF: Brief Pain Inventory— short form.

I Dichotomous variable: 0 = intermittent or brief pain, 1 = constant pain, frequency subscale of McGill Pain Questionnaire.

\# Continuous variable, HADS-A: Hospital Anxiety and Depression Scale-anxiety subscale.

†† For models 2A and 2B, no significant predictors were found in the comparison of PT2 and PT3.

Cl, confidence interval; LL, lower limit; NRS, numerical rating scale; OR, odds ratio; PT1, pain trajectory group 1 (no chronic postsurgical pain); PT2, pain trajectory group 2 (prolonged postsurgical pain); PT3, pain trajectory group 3 (chronic postsurgical pain); UL, upper limit.

Although all baseline psychological factors are common predictive factors for both PT2 and PT3 membership, presurgical fear of long-term consequences of surgery was the only variable specifically distinguishing these groups. Hence, presenting higher levels of fear related with long-term issues, such as fear of health deterioration or long-lasting surgical recovery, seems to confer a greater risk of long-lasting pain.

The set of predictive models used in this study, which include preoperative and perioperative emotion and cognitions, seem to reveal a psychological vulnerability profile, influencing PSP trajectory and ultimately the development of CPSP. This critical role of psychological factors on pain experience helps to understand the discrepancy that sometimes emerges between physical pathology, injury, and pain report. ${ }^{16,55,86,107}$ These findings are also in accordance with the gate control and neuromatrix theories of pain, ${ }^{64,65}$ as well as with the biopsychosocial approach to pain, ${ }^{37}$ which recognize pain as a multidimensional subjective experience.

Peripheral sensitization, central sensitization, and descending modulation constitute 3 interweaved processes believed to underlie CPSP. ${ }^{62}$ Augmented activation of pain pathways may stem from peripheral and/or central sensitization, although pain amplification may also arise from abnormalities in descending modulatory systems. ${ }^{72,81,109}$ These descending pathways involve endogenous opioids, serotonin, and noradrenaline, presenting both excitatory and inhibitory actions on spinal cord afferent projection neurons and could be activated by psychological factors. ${ }^{11}$ Catastrophizing, anxiety, and other negative emotions are associated with reduced effectiveness in descending pain-inhibitory systems, ${ }^{83,105}$ facilitating spinal nociception, and pain, whereas positive affect and self-regulatory skills inhibit spinal nociception and pain. ${ }^{28,39,88-90}$ The primary role of catastrophizing in pain modulation has been highlighted ${ }^{16,18,34,39,50,94}$ and suggested to be associated with diminished endogenous inhibition of pain coupled with central sensitization. ${ }^{83}$ Moreover, an association was shown between adaptive pain coping strategies and activation of descending endogenous opioid systems ${ }^{1}$ and between depressed mood and impaired endogenous inhibition of pain. ${ }^{30}$

\subsection{Practical and clinical implications}

Because chronic pain is very difficult to treat, the importance of secondary prevention is highlighted. ${ }^{38,93}$ Besides, and despite the enthusiastic and promising findings of some trials focusing on preventive analgesic approaches, ${ }^{10,12,21,29,74,96}$ there is not yet any robust evidence to support the unequivocal efficacy of systemic drugs for CPSP prevention., $17,19,43,53,60,63,86$ 
Moreover, notwithstanding the advances in knowledge about pain genetics, ${ }^{22,31,32,70,71}$ there is not, until now, sustained evidence for genetic predisposition for CPSP development ${ }^{22,48,67,86}$ that could assist in screening for pain-prone patients and in informing a personalized pain treatment protocol. This leaves room for the implementation of psychological interventions aimed at preventing CPSP. ${ }^{15,23,33,47-49,62,99}$ Indeed, present findings point to CPSP predictors that are modifiable risk factors, amenable to change or to active management, precisely through psychological interventions. Targeting them might yield adaptive and functional changes in brain pain processing, thus being an effective way to prevent CPSP development. ${ }^{34,94}$

The effectiveness of psychological interventions in pain is well established, ${ }^{36,46}$ although in the surgical field it is overdue. ${ }^{15,47,55}$ As substantial advances are being made in identifying risk factors for CPSP, the design and testing of preventive pain management strategies stemming from this evidence is warranted as well as the analysis of their cost-effectiveness. An enthusiastic and promising novelty in this field is the development and implementation of a Transitional Pain Service, a multidisciplinary program aimed at preventing and managing CPSP, which offers simultaneous psychological and pharmacological interventions. $^{20,23,42,49}$

Psychological interventions have the power to impact supraspinal mechanisms involving higher pain centers, ${ }^{62}$ influencing endogenous modulation of pain, thereby improving endogenous analgesia, similarly to mechanisms underlying pharmacological analgesia. ${ }^{34,45,46,83}$ For example, cognitive-behavioral therapy has been related to changes in brain limbic activity, which has been implicated in improvement of anxiety and potentiation of descending modulatory inhibition of pain. ${ }^{54,106}$ Increased gray matter in prefrontal and parietal brain regions linked with chronic pain, was also observed after cognitive-behavioral therapy, being associated with a decrease in pain catastrophizing. ${ }^{95}$

\subsection{Limitations and strengths}

This is a single-site and single-country study, which compromises its external validity, thus limiting the generalization of results. Sample attrition is a potential limitation, stemming from the longitudinal design, although our study showed high retention over time. Other potential limitation is the absence of a physical examination at T3 and T4, screening for inflammation or nerve injury.

The breath of presurgical measures, embracing demographic, clinical, and a thorough range of psychological variables was a strength, along with the long-term follow-up of 5 years.

\subsection{Conclusions}

This study identified risk factors that can be easily screened before and after surgery, and are amenable to intervention. The design of timely and tailored interventions for women at risk of an unfavorable PSP trajectory after hysterectomy is a key priority. Incorporating risk-targeted multidisciplinary and feasible interventions focused on CPSP prevention into surgical routine practice is an important challenge that is likely to contribute to the improvement of pain management and patient care throughout the process of surgery.

\section{Conflict of interest statement}

The authors have no conflict of interest to declare.
This work was supported by 2 grants (SFRH/BD/36368/2007 and SFRH/BPD/103529/2014) from the Portuguese Foundation of Science and Technology.

\section{Supplemental video content}

Video content associated with this article can be found at http:// links.Iww.com/PAIN/A540.

\section{Article history:}

Received 18 September 2017

Received in revised form 8 December 2017

Accepted 3 January 2018

Available online 5 February 2018

\section{References}

[1] Bandura A, O'Leary A, Taylor CB, Gauthier J, Gossard D. Perceived selfefficacy and pain control: opioid and nonopioid mechanisms. J Pers Soc Psychol 1987;53:563-71.

[2] Belfer I, Schreiber KL, Shaffer JR, Shnol H, Blaney K, Morando A, Englert D, Greco C, Brufsky A, Ahrendt G, Kehlet H, Edwards RR, Bovbjerg DH. Persistent postmastectomy pain in breast cancer survivors: analysis of clinical, demographic, and psychosocial factors. J Pain 2013;14: 1185-95.

[3] Beyaz SG, Özocak H, Ergönenç T, Palabyk O, Tuna AT, Kaya B, Erkorkmaz Ü, Akdemir N. Chronic postsurgical pain and neuropathic symptoms after abdominal hysterectomy: a silent epidemic. Medicine 2016;95:e4484.

[4] Bouhassira D, Attal N, Alchaar H, Boureau F, Brochet B, Bruxelle J, Cunin G, Fermanian J, Ginies P, Grun-Overdyking A, Jafari-Schluepi H, Lantéri-Minet M, Laurent B, Mick G, Serrie A, Valade D, Vicaut E. Comparison of pain syndromes associated with nervous or somatic lesions and development of a new neuropathic pain diagnostic questionnaire (DN4). PAIN 2005;114:29-36.

[5] Bouhassira D, Lanteri-Minet M, Attal N, Laurent B, Touboul C. Prevalence of chronic pain with neuropathic characteristics in the general population. PAIN 2008;136:380-7.

[6] Brandsborg B, Dueholm M, Nikolajsen L, Kehlet H, Jensen TS. A prospective study of risk factors for pain persisting 4 months after hysterectomy. Clin J Pain 2009;25:263-8.

[7] Brandsborg B, Nikolajsen L, Hansen CT, Kehlet H, Jensen TS. Risk factors for chronic pain after hysterectomy: a nationwide questionnaire and database study. Anesthesiology 2007;106:1003-12.

[8] Brandsborg B, Nikolajsen L, Kehlet H, Jensen TS. Chronic pain after hysterectomy. Acta Anaesthesiol Scand 2008;52:327-31.

[9] Breivik $\mathrm{H}$. Ketamine has anti-hyperalgesic effects and relieves acute pain, but does not prevent persistent postoperative pain (PPP). Scand J Pain 2015;7:40-1.

[10] Burke SM, Shorten GD. Perioperative pregabalin improves pain and functional outcomes 3 months after lumbar discectomy. Anesth Analg 2010;110:1180-5.

[11] Bushnell MC, Čeko M, Low LA. Cognitive and emotional control of pain and its disruption in chronic pain. Nat Rev Neurosci 2013;14:502-11.

[12] Buvanendran A, Kroin JS, Della Valle CJ, Kari M, Moric M, Tuman KJ. Perioperative oral pregabalin reduces chronic pain after total knee arthroplasty: a prospective, randomized, controlled trial. Anesth Analg 2010;110:199-207.

[13] Bruce J, Quinlan J. Chronic post surgical pain. Rev pain 2011;5:23-9.

[14] Bruce J, Thornton AJ, Powell R, Johnston M, Wells M, Heyes SD, Thompson AM, Smith WC, Chambers WA; Scott NW on Behalf of the Recovery Study Group. Psychological, surgical and sociodemographic predictors of pain outcomes after breast cancer surgery: a populationbased cohort study. PAIN 2014;155:232-43.

[15] Burns JW, Moric M. Psychosocial factors appear to predict postoperative pain: Interesting, but how can such information be used to reduce risk? Tech Reg Anesth Pain Manag 2011;15:90-9.

[16] Campbell CM, Edwards RR. Mind-body interactions in pain: the neurophysiology of anxious and catastrophic pain-related thoughts. Translational Res 2009;153:97-101.

[17] Chaparro LE, Smith SA, Moore RA, Wiffen PJ, Gilron I. Pharmacotherapy for the prevention of chronic pain after surgery in adults. Cochrane Database Syst Rev 2013;CD008307. doi: 10.1002/ 14651858.CD008307.pub2. 
[18] Chehadi O, Suchan B, Konietzny K, Köster O, Schmidt-Wilcke T, Hasenbring Ml. Gray matter alteration associated with pain catastrophizing in patients 6 months after lumbar disk surgery: a voxel-based morphometry study. Pain Rep 2017;00:e617.

[19] Chelly JE. Pregabalin effective for the prevention of chronic postsurgical pain: really? Anesth Analg 2013;116:507-8.

[20] Clarke H. Transitional pain medicine: novel pharmacological treatments for the management of moderate to severe postsurgical pain. Expert Rev Clin Pharmacol 2016;9:345-9.

[21] Clarke H, Bonin RP, Orser BA, Englesakis M, Wijeysundera DN, Katz J. The prevention of chronic postsurgical pain using gabapentin and pregabalin: a combined systematic review and meta-analysis. Anesth Analg 2012;115:428-42.

[22] Clarke H, Katz J, Flor H, Rietschel M, Diehl SR, Seltzer ZE. Genetics of chronic post-surgical pain: a crucial step toward personal pain medicine. Can J Anaesth 2015;62:294-303.

[23] Clarke H, Poon M, Weinrib A, Katznelson R, Wentlandt K, Katz J. Preventive analgesia and novel strategies for the prevention of chronic post-surgical pain. Drugs 2015;75:339-51.

[24] Cleeland C, Ryan KM. Pain assessment: global use of the Brief Pain Inventory. Ann Acad Med 1994;23:129-38.

[25] Cohen J. Statistical power analysis for the behavioural sciences. 2nd ed. Hillsdale: Lawrence Erlbaum Associates, 1998.

[26] Crombie IK, Davies HT, Macrae WA. Cut and thrust: antecedent surgery and trauma among patients attending a chronic pain clinic. PAIN 1998; 76:167-71.

[27] Cronbach LJ. Coefficient alpha and the internal structure of tests. Psychometrika 1951:16:297-334.

[28] Darnall BD. Pain psychology and pain catastrophizing in the perioperative setting a review of impacts, interventions, and unmet needs. Hand Clin 2016;32:33-9.

[29] De Kock M, Lavand'homme P, Waterloos H. "Balanced analgesia" in the perioperative period: is there a place for ketamine? PAIN 2001;92: 373-80.

[30] De Souza JB, Potvin S, Goffaux P, Charest J, Marchand S. The deficit of pain inhibition in fibromyalgia is more pronounced in patients with comorbid depressive symptoms. Clin J Pain 2009;25:123-7.

[31] Diatchenko L, Anderson AD, Slade GD, Fillingim RB, Shabalina SA, Higgins TJ, Sama S, Belfer I, Goldman D, Max MB, Weir BS, Maixner W. Three major haplotypes of the beta2 adrenergic receptor define psychological profile, blood pressure, and the risk for development of a common musculoskeletal pain disorder. Am J Med Genet B Neuropsychiatr Genet 2006;141B:449-62.

[32] Diatchenko L, Slade GD, Nackley AG, Bhalang K, Sigurdsson A, Belfer I, Goldman D, XU K, Shabalina SA, Max D, Makarov SS, Maixner W. Genetic basis for individual variations in pain perception and the development of a chronic pain condition. Hum Mol Genet 2005;14: 135-43.

[33] Dworkin RH, McDermott MP, Raja SN. Preventing chronic postsurgical pain: how much of a difference makes a difference? Anesthesiology 2010;112:516-8.

[34] Edwards RR, Dworkin RH, Sullivan MD, Turk DC, Wasan AD. The role of psychosocial processes in the development and maintenance of chronic pain. J Pain 2016;17:T70-92.

[35] Fletcher D, Stamer UM, Pogatzki-Zahn E, Zaslansky R, Tanase NV, Perruchoud C, Kranke P, Komann M, Lehman T; euCPSP Group for the Clinical Trial Network Group of the European Society of Anaesthesiology, Meissner W. Chronic postsurgical pain in Europe: an observational study. Eur J Anaesthesiol 2015;32:725-34.

[36] Gatchel RJ, Okifuji A. Evidence-based scientific data documenting the treatment and cost-effectiveness of comprehensive pain programs for chronic nonmalignant pain. J Pain 2006;7:779-93

[37] Gatchel RJ, Peng YB, Peters ML, Fuchs PN, Turk DC. The biopsychosocial approach to chronic pain: scientific advances and future directions. Psychol Bull 2007;133:581-624.

[38] Gilron I, Kehlet H. Prevention of chronic pain after surgery: new insights for future research and patient care. Can J Anaesth 2014;61:101-11.

[39] Goodin BR, McGuire L, Allshouse M, Stapleton L, Haythornthwaite JA, Burns N, Mayes LA, Edwards RR. Associations between catastrophizing and endogenous pain-inhibitory processes: sex differences. J Pain 2009;10:180-90.

[40] Hetmann F, Kongsgaard UE, Sandvik L, Schou-Bredal I. Prevalence and predictors of persistent post-surgical pain 12 months after thoracotomy. Acta Anaesthesiol Scand 2015;59:740-48.

[41] Hinrichs-Rocker A, Schulz K, Järvinen I, Lefering R, Simanski C, Neugebauer EA. Psychosocial predictors and correlates for chronic post-surgical pain (CPSP)—a systematic review. Eur J Pain 2009;13: 719-30.
[42] Huang A, Katz J, Clarke H. Ensuring safe prescribing of controlled substances for pain following surgery by developing a transitional pain service. Pain Manag 2015;5:97-105.

[43] Humble SR, Dalton AJ, Li L. A systematic review of therapeutic interventions to reduce acute and chronic post-surgical pain after amputation, thoracotomy or mastectomy. Eur J Pain 2015;19:451-65.

[44] Jackson T, Tian P, Wang Y, Lezzi T, Xie W. Towards identifying moderators of associations between pre-surgery emotional distress and post-operative pain outcomes: a meta-analysis of longitudinal studies. J Pain 2016;17:874-88.

[45] Jensen MP. A neuropsychological model of pain: research and clinical implications. J Pain 2010;11:2-12.

[46] Jensen MP, Turk DC. Contributions of psychology to the understanding and treatment of people with chronic pain: why it matters to ALL psychologists. Am Psychol 2014;69:105-18.

[47] Kalso E. IV. Persistent post-surgery pain: research agenda for mechanisms, prevention, and treatment. Br J Anaesth 2013;111:9-12.

[48] Katz J, Seltzer Z. Transition from acute to chronic postsurgical pain: risk factors and protective factors. Expert Rev Neurother 2009;9:723-44.

[49] Katz J, Weinrib A, Fashler SR, Katznelzon R, Shah BR, Ladak SS, Jiang Jiao, Li Q, McMillan K, Mina DS, Wentlandt K, McRae K, Tamir D, Lyn S, de Perrot M, Rao V, Grant D, Roche-Nagle G, Cleary S, Hofer S, Gilbert R, Wijeysundera D, Ritvo P, Janmohamed T, O'Leary G, Clarke H. The Toronto General Hospital Transitional Pain Service: development and implementation of a multidisciplinary program to prevent chronic postsurgical pain. J Pain Res 2015;8:1-8.

[50] Khan RS, Ahmed K, Blakewayet E, Skapinakis P, Nihoyannopoulos L, Macleod K, Sevdalis N, Ashrafian H. Platt M, Darzi A, Athanasiou T. Catastrophizing: a predictive factor for postoperative pain. Am J Surg 2011;201:122-31.

[51] Kehlet $H$, Jensen TS, Woolf $C$. Persistent postsurgical pain: risk factors and prevention. Lancet 2006;367:1618-25.

[52] Kehlet H, Rathmell JP. Persistent postsurgical pain: the path forward through better design of clinical studies. Anesthesiology 2010;112:514-5.

[53] Klatt E, Zumbrunn T, Bandschapp O, Girard T, Ruppen W. Intra- and postoperative intravenous ketamine does not prevent chronic pain: a systematic review and meta-analysis. Scand J Pain 2015;7:42-54.

[54] Lackner JM, Lou CM, Mertz HR, Wack DS, Katz LA, Krasner SS, Firth R, Mahl TC, Lockwood AH. Cognitive therapy for irritable bowel syndrome is associated with reduced limbic activity, Gl symptoms, and anxiety. Behav Res Ther 2006;44:621-38.

[55] Lavand'homme P. Transition from acute to chronic pain after surgery. PAIN 2017;158:S50-4.

[56] Lewis GN, Rice DA, McNair PJ, Kluger M. Predictors of persistent pain after total knee arthroplasty: a systematic review and meta-analysis. $\mathrm{Br} \mathrm{J}$ Anaesth 2015;114:551-61.

[57] Llewellyn CD, McGurk M, Weinman K. Illness and treatment beliefs in head and neck cancer: is Leventhal's common sense model a useful framework for determining changes in outcomes over time? J Psychosom Res 2007;63:17-26.

[58] Lunn TH, Gaarn-Larsen L, Kehlet H. Prediction of postoperative pain by preoperative pain response to heat stimulation in total knee arthroplasty. PAIN 2013;154:1878-85.

[59] Macrae WA. Chronic postsurgical pain: 10 years on. Br J Anaesth 2008; 101:77-86.

[60] Martinez V, Pichard X, Fletcher D. Perioperative pregabalin administration does not prevent chronic postoperative pain: systematic review with a meta-analysis of randomized trials. PAIN 2017; 158:775-83.

[61] Masselin-Dubois A, Attal N, Fletcher D, Jayr C, Albi A, Fermanian J, Bouhassira D, Baudic S. Are psychological predictors of chronic postsurgical pain dependent on the surgical model? A comparison of total knee arthroplasty and breast surgery for cancer. J Pain 2013;14: 854-64.

[62] McGreevy K, Bottros MM, Srinivasa NR. Preventing chronic pain following acute pain: risk factors, preventive strategies, and their efficacy. Eur J Pain Suppl 2011;5:365-76.

[63] McNicol ED, Schumann R, Haroutounian S. A systematic review and meta-analysis of ketamine for the prevention of persistent post-surgical pain. Acta Anaesthesiol Scand 2014;58:1199-213.

[64] Melzack R. From the gate to the neuromatrix. PAIN 1999;6(suppl): S121-26.

[65] Melzack R. Pain-an overview. Acta Anaesthesiol Scand 1999;43: 880-4.

[66] Melzack R. The McGill Pain Questionnaire: major properties and scoring methods. PAIN 1975;1:277-99.

[67] Montes A, Roca G, Sabate S, Lao JI, Navarro A, Cantillo J, Canet J. Genetic and clinical factors associated with chronic postsurgical pain 
after hernia repair, hysterectomy, and thoracotomy: a two-year multicenter cohort study. Anesthesiology 2015;122:1123-41.

[68] Moss-Morris R, Weinman J, Petrie KJ, Horne R, Cameron LD, Buick D. The Revised IIIness Perception Questionnaire (IPQ-R). Psychol Health 2002;17:1-16.

[69] Nikolajsen L. Perioperative interventions for the reduction of chronic postsurgical pain. PAIN 2017;158:769-70.

[70] Nissenbaum J, Devor M, Seltzer ZE, Gebauer M, Michaelis M, Tal M, Dorfman R, Abitbul-Yarkoni M, Lu Y, Elahipanah T, delCanho S, Minert A, Fried K, Persson AK, Shpigler H, Shabo E, Yakir B, Pisanté A, Darvasi A. Susceptibility to chronic pain following nerve injury is genetically affected by CACNG2. Genome Res 2010;20:1180-90.

[71] Oertel B, Lotsch J. Genetic mutations that prevent pain: implications for future pain medication. Pharmacogenomics 2008;9:179-94.

[72] Ossipov MH, Dussor GO, Porreca F. Central modulation of pain. J Clin Invest 2010;120:3779-87.

[73] Perkins FM, Kehlet H. Chronic pain as an outcome of surgery. A review of predictive factors. Anesthesiology 2000;93:1123-33.

[74] Pesonen A, Suojaranta-Ylinen R, Hammarén E, Kontinen VK, Raivio P, Tarkkila P, Rosenberg PH. Pregabalin has an opioid sparing effect in elderly patients after cardiac surgery: a randomized placebo-controlled trial. Br J Anaesth 2011;106:873-81.

[75] Pinto PR, Mclntyre T, Almeida A, Araújo-Soares V. The mediating role of pain catastrophizing in the relationship between presurgical anxiety and acute postsurgical pain after hysterectomy. PAIN 2012;153:218-26.

[76] Pinto PR, Mclntyre T, Araújo-Soares V, Costa P, Almeida A. Differential predictors of acute post-surgical pain intensity after abdominal hysterectomy and major joint arthroplasty. Ann Behav Med 2015;49:384-97.

[77] Pinto PR, Mclntyre T, Ferrero R, Almeida A, Araújo-Soares V. Risk factors for moderate and severe persistent pain in patients undergoing total knee and hip arthroplasty: a prospective predictive study. PLoS One 2013;8:e73917.

[78] Pinto PR, Mclntyre T, Fonseca C, Almeida A, Araújo-Soares V. Pre- and post-surgical factors that predict the provision of rescue analgesia following hysterectomy. Eur J Pain 2013;17:423-33.

[79] Pinto PR, Mclntyre T, Nogueira-Silva C, Almeida A, Araújo-Soares V. Risk factors for persistent postsurgical pain in women undergoing hysterectomy due to benign causes: a prospective predictive study. J Pain 2012;13:1045-57.

[80] Pokkinen SM, Nieminen K, Yli-Hankala A, Kalliomäki ML. Persistent posthysterectomy pain: a prospective, observational study. Eur J Anaesth 2015;32:718-24.

[81] Porreca F, Ossipov MH, Gebhart GF. Chronic pain and medullary descending facilitation. Trends Neurosci 2002;25:319-25.

[82] Powell R, Johnston M, Smith WC, King PM, Chambers WA, Krukowski Z, McKee L, Bruce J. Psychological risk factors for chronic post-surgical pain after inguinal hernia repair surgery: a prospective cohort study. Eur J Pain 2012;16:600-10.

[83] Quartana PJ, Campbell CM, Edwards RR. Pain catastrophizing: a critical review. Expert Rev Neurother 2009;9:745-58.

[84] Rea LM, Parker RA. Designing and conducting survey research. San Francisco: Jossey-Bass, 1992.

[85] Recker DC, Perry PM. Postsurgical pain syndromes: chronic pain after hysterectomy and cesarean section. Tech Reg Anesth Pain Manag 2011;15:133-9.

[86] Richebé P, Julien M, Brulotte V. Potential strategies for preventing chronic postoperative pain: a practical approach: continuing professional development. Can J Anesth 2015;62:1329-41.

[87] Riley JL, Robinson ME. CSQ: five factors or fiction? Clin J Pain 1997; 13: 156-62.

[88] Rhudy JL, Williams AE, McCabe K, Nguyen MA, Rambo P. Affective modulation of nociception at spinal and supraspinal levels. Psychophysiology 2005;42:579-87.

[89] Rhudy JL, Williams AE, McCabe KM, Rambo PL, Russell JL. Emotional modulation of spinal nociception and pain: the impact of predictable noxious stimulation. PAIN 2006;126:221-33.

[90] Rhudy JL, Williams AE, McCabe KM, Russell JL, Maynard LJ. Emotional control of nociceptive reactions (ECON): do affective valence and arousal play a role? PAIN 2008;136:250-61.
[91] Scharloo M, de Jong RJ, Langeveld TP, Velzen-Verkaik E, Akker MM, Kaptein AA. Quality of life and illness perceptions in patients with recently diagnosed head and neck cancer. Head Neck 2005;27:857-63.

[92] Scheier MF, Carver CS, Bridges MW. Distinguishing optimism from neuroticism (and trait anxiety, self-mastery, and self-esteem): a reevaluation of the Life Orientation Test. J Pers Soc Psychol 1994; 67:1063-78.

[93] Schug SA, Pogatzki-Zahn EM. Chronic pain after surgery or injury. PAIN 2011;19:1-5

[94] Seminowicz DA, Davis KD. Cortical responses to pain in healthy individuals depends on pain catastrophizing. PAIN 2006;120:297-306.

[95] Seminowicz DA, Shpaner M, Keaser ML, Krauthamer GM, Mantegna J, Dumas JA, Newhouse PA, Filippi CG, Keefe FJ, Naylor MR. Cognitivebehavioral therapy increases prefrontal cortex gray matter in patients with chronic pain. J Pain 2013;14:1573-84.

[96] Sen H, Sizlan A, Yanarates O, Emirkadi H, Ozkan S, Dagli G, Turan A. A comparison of gabapentin and ketamine in acute and chronic pain after hysterectomy. Anesth Analg 2009;109:1645-50.

[97] Sørensen J, Kjeldsen J, Kugathasan P, Lunde S, Andersen E, Skov M, Arendt-Nielsen L. The risk of developing postoperative chronic pain after abdominal and robot-assisted laparoscopic hysterectomy: a crosssectional study. J Gynecol Surg 2015;31:198-204.

[98] Thakar R, Ayers S, Clarkson P, Stanton S, Manyonda I. Outcomes after total versus subtotal abdominal hysterectomy. N Engl J Med 2002;347: 1318-25.

[99] Theunissen M, Peters ML, Bruce J, Gramke HF, Marcus MA. Preoperative anxiety and catastrophizing: a systematic review and meta-analysis of the association with chronic postsurgical pain. Clin $J$ Pain 2012;28:819-41.

[100] Theunissen M, Peters ML, Schepers J, Maas JW, Tournois F, van Suijlekom HA, Gramke HF, Marcus MA. Recovery 3 and 12 months after hysterectomy. epidemiology and predictors of chronic pain, physical functioning, and global surgical recovery. Medicine 2016;95: e3980.

[101] Theunissen M, Peters ML, Schouten EG, Fiddelers AA, Willemsen MGA, Pinto PR, Gramke HF, Marcus MA. Validation of the Surgical Fear Questionnaire in adult patients waiting for elective surgery. PLoS One 2014;9:e100225.

[102] Treede RD, Rief W, Barke A, Aziz Q, Bennett MI, Benoliel R, Cohen M, Evers S, Finnerup NB, First MB, Giamberardino MA, Kaasa S, Kosec E, Lavand'homme P, Nicholas M, Perrot S, Scholz J, Schug S, Smith BH, Svensson P, Vlaeyen J, Wang S. A classification of chronic pain for ICD11. PAIN 2015;156:1003-7.

[103] VanDenKerkhof EG, Hopman WM, Goldstein DH, Wilson RA, Towheed TE, Lam M, Harrison MB, Reitsma ML, Johnston SL, James D, Gilron I. Impact of perioperative pain intensity, pain qualities, and opioid use on chronic pain after surgery: a prospective cohort study. Reg Anesth Pain Med 2012;37:19-27.

[104] VanDenKerkhof EG, Peters ML, Bruce J. Chronic pain after surgery: time for standardization? A framework to establish core risk factor and outcome domains for epidemiological studies. Clin J Pain 2013;29:2-8.

[105] van Wijk G, Veldhuijzen DS. Perspective on diffuse noxious inhibitory controls as a model of endogenous pain modulation in clinical pain syndromes. J Pain 2010;11:408-19.

[106] Wilder-Smith CH, Schindler D, Lovblad K, Redmond SM, Nirkko A. Brain functional magnetic resonance imaging of rectal pain and activation of endogenous inhibitory mechanisms in irritable bowel syndrome patient subgroups and healthy controls. Gut 2004;53: 1595-601.

[107] Wildgaard K, Ringsted TK, Hansen HJ, Petersen RH, Werner MU, Kehlet $\mathrm{H}$. Quantitative sensory testing of persistent pain after videoassisted thoracic surgery lobectomy. Br J Anaesth 2012;108:126-33.

[108] Wylde V, Hewlett S, Learmonth ID, Dieppe P. Persistent pain after joint replacement: prevalence, sensory qualities, and postoperative determinants. PAIN 2011;152:566-72.

[109] Zhuo M. Descending facilitation: from basic science to the treatment of chronic pain. Mol Pain 2017;13:1-12.

[110] Zigmond AS, Snaith RP. The Hospital Anxiety and Depression Scale. Acta Psychiatrica Scand 1983;67:361-70. 